\title{
EVALUACIÓN DE LAS NECESIDADES DE LOS PACIENTES RESPECTO A LA INFORMACIÓN Y LOS CUIDADOS MÉDICOS TRAS EL DIAGNÓSTICO DE CÁNCER
}

\section{ASSESSING THE NEEDS OF PATIENTS ABOUT INFORMATION AND MEDICAL CARE AFTER DIAGNOSIS OF CANCER}

Ernesto Gil Deza', Daniela Gercovich², Eduardo Morgenfeld', Flavio Tognelli', Gastón Martín Reinas ${ }^{1}$, Pablo López ${ }^{2}$, Mónica Montiel' ${ }^{1}$, Carlos García Gerardi ${ }^{1}$, Edgardo Rivarola $^{7}$ y Felipe Gustavo Gercovich?

1 Instituto Oncológico Henry Moore. Buenos Aires, Argentina

2 Instituto William Osler. Buenos Aires, Argentina

Resumen

Objetivo: Estudiar las necesidades de los pacientes oncológicos sobre la información referida a su enfermedad y los cuidados al final de la vida.

Métodos: 820 pacientes participaron de una encuesta sobre diagnóstico, pronóstico, eficacia y toxicidad de los tratamientos, así como también sobre cuidados paliativos, investigación clínica, apoyo psicológico, cuidados espirituales y deseos del final de la vida.

Resultados: 695 pacientes conocen su diagnóstico, especialmente quienes tenían un mayor nivel educativo $y$ un rango etario de 50-70 años. Seiscientos setenta y cuatro pacientes deseaban conocer su pronóstico, estando más predispuestos a conocerlo quienes tenían educación secundaria y superior.

La mayoría (562 pacientes) deseaba conocer todo con respecto a la utilidad de los tratamientos, al igual que con respecto a la toxicidad (443) y valoraría contar con información sobre cuidados paliativos e investigación. Además consideraba la internación hospitalaria como el mejor lugar para morir (331).

Las mujeres se mostraron más propensas a recibir ayuda psicológica y espiritual.

Las condiciones más valoradas en el médico fueron: conocimiento $(52,7 \%)$, Honestidad $(45,5 \%)$ y Humanidad $(44,3 \%)$.
Abstract

Objective: To study and understand the needs for informations of the patients in our environment, analyzing specifically the needs for information about their illness and care at the end of life after being diagnosed with cancer.

Methods: 820 patients in a survey about diagnosis, prognosis, treatment efficacy and toxicity, as well as palliative care, clinical research, psychological support, spiritual care and wishes of the end of life.

Results: Patients majority (695) was aware of their diagnosis, knowing more who had higher education and an age range of 5070 years. Six hundred seventy-four patients wanted to know their prognosis, being more likely to know those who had secondary and higher education.

The majority (562 patients) wanted to know everything about the treatment utility, as well as with respect to toxicity (443) and would value to have information on palliative care and research. Hospitalization also considered as the best place to die (331).

Women were more likely to receive psychological and spiritual help.

The most valued physician conditions were knowledge $(52.7 \%)$, honesty $(45.5 \%)$ and Humanities (44.3\%).

\section{Correspondencia:}


Un $82,3 \%$ de los pacientes valoraron positivamente la encuesta, mientras que el $58 \%$ la consideró útil. Sin embargo un $23,7 \%$ la recomendaría.

Conclusiones: El estudio representa, en nuestro conocimiento, el primer intento en nuestro medio por identificar las necesidades y los deseos de los pacientes por conocer cuestiones vinculadas con su enfermedad. En este sentido hemos observado una búsqueda de participación activa en las decisiones vinculadas al manejo de la información y los tratamientos por parte de los pacientes.

Palabras clave: Necesidades de los pacientes, oncología, comunicación médico paciente, encuesta.
The $82.3 \%$ of patients appreciated the survey, while $58 \%$ considered it useful. However, $23.7 \%$ would recommend it.

Conclusions: This study represents, to our knowledge, the first attempt in our country to identify the needs and wishes of patients to know about issues related to their illness. We have observed a search for active participation in decisions related to information management and treatments by patients.

Key words: Patient's needs, oncology, physician-patient communication, survey.

\section{INTRODUCCIÓN}

Durante los últimos años se ha incrementado el interés por conocer las preferencias de los pacientes con respecto a la información que desean recibir de los médicos sobre su enfermedad y su pronóstico. Esto surge en oposición a una serie de prejuicios que han condicionado el accionar de los profesionales de la salud a lo largo de la historia de la medicina.

En primer lugar, el paternalismo médico sostenía la idea de que la verdad puede ser más dañina que el engaño y daba al médico la posibilidad de poseer todo el saber y determinar qué informar al paciente y qué no. Es decir, el médico no solamente tenía el conocimiento técnico-científico sino que decidía qué información brindar con respecto al diagnóstico, el pronóstico y el tratamiento, así como también era quien estimaba cuáles eran los deseos y las necesidades de los pacientes de modo unilateral.

Posteriormente, particularmente en nuestro medio, el paternalismo médico fue reemplazado por el paternalismo cultural anglosajón, en donde la información médica y las relaciones interpersonales se extrapolan automáticamente de un ámbito cultural al otro sin validación alguna.
Esta cuestión hace imprescindible que se diseñen estudios tendientes a conocer en mayor profundidad las características de los pacientes de nuestro medio con las particularidades socio-culturales que los caracterizan.

En la actualidad, lo que conocemos por estudios previos en otras poblaciones, es que habitualmente los médicos son renuentes a brindar información sobre pronósticos negativos ${ }^{(1)}$ por miedo a disminuir la esperanza del paciente ${ }^{(2)}$. Es importante señalar que tanto los médicos como los pacientes suelen estar ambivalentes con respecto a hablar de la muerte y frecuentemente tienden a evitar esta conversación $^{(3-8)}$. Posiblemente una de las razones que expliquen esta ambivalencia se relacione con que los médicos no cuentan con la información suficiente para poder sopesar los riesgos y beneficios de la conversación al final de la vida ${ }^{(9)}$.

Sumado a lo anterior, en portales web específicos no se encuentra información detallada con respecto al pronóstico, datos de sobrevida, opciones de cuidado paliativo ni datos de referencias de hospitales especializados $^{(10)}$.

Por las razones expuestas los pacientes conocen poco acerca de su pronóstico y 
el efecto que el tratamiento tendrá sobre su enfermedad. Esto contrasta con el hecho de que los pacientes prefieren obtener información honesta, comprensible y clara sobre su enfermedad ${ }^{(10-12)}$.

Dado que en la actualidad carecemos de estudios que evalúen lo descripto anteriormente en nuestra población, hemos diseñado el presente trabajo con el objetivo de estudiar y comprender las necesidades de nuestros pacientes en nuestro medio, analizando las necesidades con respecto a la información sobre su enfermedad y los cuidados al final de la vida luego del diagnóstico de cáncer.

\section{MÉTODO}

\section{Diseño}

El presente es un estudio descriptivo observacional mediante encuesta.

\section{Participantes}

Se seleccionaron consecutivamente 1100 pacientes con diagnóstico de cáncer en seguimiento o tratamiento oncológico en nuestro instituto que aceptaron participar de una encuesta anónima orientada a conocer sus deseos y necesidades sobre la información y los cuidados médicos al final de la vida.

\section{Instrumento}

Se diseñó una encuesta ad hoc de manera interdisciplinaria que consignó: edad, sexo, si estaba en pareja, si tenía hijos y nivel educativo como variables de estudio y se analizaron los deseos con respecto a la información diagnóstica, pronóstica, utilidad y toxicidad de los tratamientos, interés en tópicos de investigación, cuidados paliativos, soporte psicológico, soporte espiritual y decisiones sobre el final de la vida: selección de la persona que represente su voluntad en caso de inconsciencia y lugar en el que desearía fallecer en caso de ser un paciente terminal (Ver el anexo).

El instrumento y el estudio fueron presentados al Comité de Docencia e Investigación y fueron aprobados para su implementación en el instituto.

\section{Análisis de datos}

Se calcularon los estadísticos descriptivos de la muestra. Para evaluar posibles asociaciones entre variables categóricas se ejecutó la prueba de Chi cuadrado. Se utilizó el paquete estadístico IBM SPSS 19.0

\section{RESULTADOS}

\section{Datos demográficos}

La encuesta fue ofrecida a 1003 pacientes, de los cuales aceptaron participar 820 .

Los datos demográficos se detallan en la tabla 1 y la distribución de diagnósticos en la tabla 2.

\section{Conocimiento del diagnóstico}

Sobre el total de la muestra, 695 sujetos conocen el diagnóstico (1 no respondió a esta pregunta). En esta variable se observó una clara diferencia significativa según la variable sexo, dado que una mayor proporción de mujeres afirmaron conocer el diagnóstico en comparación con los hombres $\left(X^{2}=5,828 ; p=0,016\right)$ (ver la tabla 3 ). En referencia a la pregunta relacionada con la medida en que conocían el motivo de consulta, 113 respondieron que conocían poco y 430 mucho (277 no la respondieron). En este punto se encontró un mayor conocimiento por parte de los participantes que presentaban un nivel educativo secundario o superior $\left(X^{2}=33,706 ; \mathrm{p}<0,001\right)$ y un rango etario de 50-70 años $\left(X^{2}=26,181 ; p<0,001\right)$ (ver tablas 4 y 5 ). 
Tabla 1. Datos demográficos de la muestra encuestada ( $\mathbf{N}=\mathbf{8 2 0})$

\begin{tabular}{lcc}
\hline & $\mathrm{N}$ & $\%$ \\
\cline { 2 - 3 } Género & 332 & 40,5 \\
Femenino & 207 & 25,2 \\
Masculino & 281 & 34,3 \\
NS/NC & & \\
Nivel Educativo & 186 & 22,7 \\
Primario completo/incompleto & 348 & 42,4 \\
Secundario completo/incompleto & 213 & 26 \\
Superior completo/incompleto & 73 & 8,9 \\
NS/NC & & \\
Ocupación & 380 & 46,3 \\
Trabajadores activos & 239 & 29,1 \\
Jubilados & 112 & 13,7 \\
Desocupados & 89 & 10,9 \\
NS/NC & & \\
\hline
\end{tabular}

Tabla 2. Frecuencias de Diagnóstico Oncológico $(\mathbf{N}=\mathbf{8 2 0})$

\begin{tabular}{lcc}
\hline & $\mathrm{N}$ & $\%$ \\
\hline Mama & 263 & 32,07 \\
Próstata & 68 & 8,29 \\
Colorrectal & 63 & 7,68 \\
Riñón & 23 & 2,8 \\
LNH & 21 & 2,56 \\
Pulmón & 19 & 2,31 \\
Vejiga & 18 & 2,2 \\
Cabeza y cuello & 16 & 1,95 \\
Melanoma & 15 & 1,83 \\
Cervix & 14 & 1,71 \\
Ovario & 13 & 1,59 \\
Síndromes mielodisplásicos & 12 & 1,46 \\
Otros & 53 & 6,46 \\
Perdidos & 222 & 27,07 \\
\hline
\end{tabular}

Tabla 3. Tabla de contingencia.

Conocimiento del diagnóstico según sexo

\begin{tabular}{llccccc}
\hline & & \multicolumn{4}{c}{ Conoce DX } \\
\cline { 3 - 6 } & & No & $\%$ & Si & $\%$ & Total \\
\hline Sexo & Femenino & 30 & 47,62 & 301 & 63,37 & 331 \\
& Masculino & 33 & 52,38 & 174 & 36,63 & 207 \\
\multirow{2}{*}{ Total } & & 63 & 100 & 475 & 100 & 538 \\
\hline
\end{tabular}




\section{Tabla 4. Tabla de contingencia: Medida en la que conoce el diagnóstico según nivel educativo}

\begin{tabular}{llccccc}
\hline & & \multicolumn{4}{c}{ Medida de conocimiento } & \multirow{2}{*}{ Total } \\
\cline { 3 - 7 } & & Poco & $\%$ & Mucho & $\%$ & \% \\
\hline \multirow{2}{*}{ Educación } & Primario & 49 & 46,23 & 98 & 23,67 & 147 \\
\cline { 2 - 7 } & Secundario & 50 & 47,17 & 191 & 46,13 & 241 \\
\cline { 2 - 7 } & Superior/Universitario & 7 & 7,7 & 125 & 30,19 & 132 \\
\hline Total & 106 & 100 & 414 & 100 & 520 \\
\hline
\end{tabular}

Tabla 5. Tabla de contingencia: Conocimiento del diagnóstico según edad

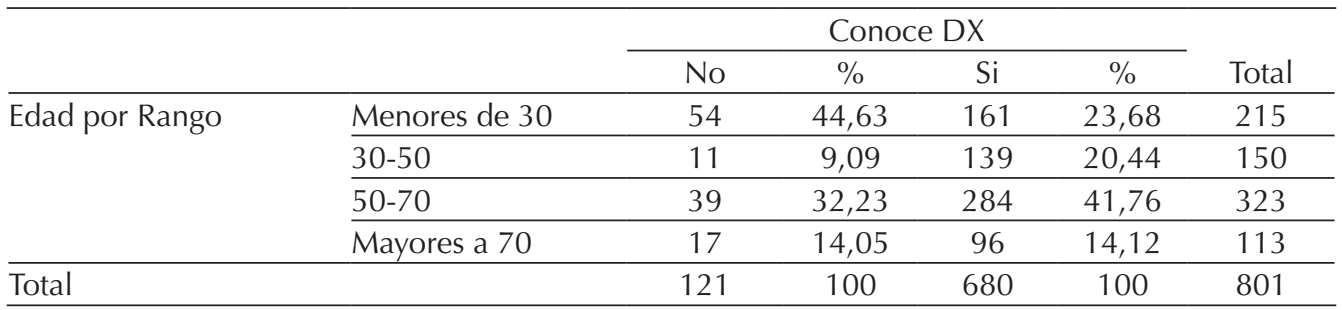

Segmentando por estadio de la enfermedad, se observó una diferencia estadísticamente significativa con respecto a conocer el diagnóstico, observando una menor tendencia a conocerlo en los pacientes de estadios I y II $\left(X^{2}=8,297 ; \mathrm{p}<0,004\right)$ (ver tabla 6).

Setecientos treinta y tres pacientes autorizarían al equipo tratante a brindar información a algún familiar y acceso a la historia clínica $(89,4 \%)$.

\section{Conocimiento del pronóstico}

Del total de la muestra 674 participantes deseaban conocer el pronóstico más allá de si era incurable y/o malo, 38 deseaban conocerlo siempre que fuera curable, 26 no querían conocerlo en algunas condiciones y 13 no querían conocerlo en absoluto (68 sujetos decidieron no responder a esta pregunta).

Cuando analizamos el interés por el pronóstico surgió claramente una diferencia en cuanto a la educación $\left(X^{2}=19,955\right.$; $p=0,003)$. Las personas con educación secundaria y superior se mostraron más predispuestas a conocer el pronóstico de la enfermedad (ver tabla 7).

Con respecto a la utilidad de los tratamientos, 20 pacientes $(2,4 \%)$ no deseaban conocer nada al respecto, 159 (19,4\%) dejarían en manos del médico la información a brindar y 562 (68,5\%) deseaban conocer todo. El resto de los participantes deseaba conocer algún aspecto de la utilidad (curación o posibilidades de curación).

Con respecto al estadio de la enfermedad, no se encontraron diferencias significativas en cuanto al interés por conocer el pronóstico.

Otros intereses vinculados con los tratamientos

En cuanto a la toxicidad de los tratamientos, 28 pacientes $(3,4 \%)$ refirieron no querer conocer nada al respecto, 194 $(23,7 \%)$ dejaban la información en manos del médico, 443 (54\%) querían conocer 
Tabla 6. Tabla de contingencia: Conocimiento del diagnóstico según estadio de la enfermedad

\begin{tabular}{|c|c|c|c|c|c|}
\hline & \multicolumn{4}{|c|}{ Conoce DX } & \multirow[b]{2}{*}{ Tota } \\
\hline & No & $\%$ & $\mathrm{Si}$ & $\%$ & \\
\hline Estadios I y II & 45 & 72,58 & 216 & 53,07 & 261 \\
\hline Estadios III y IV & 17 & 27,42 & 191 & 46,93 & 208 \\
\hline Total & 62 & 100 & 407 & 100 & 469 \\
\hline
\end{tabular}

Tabla 7. Tabla de contingencia: Conocimiento del pronóstico según educación

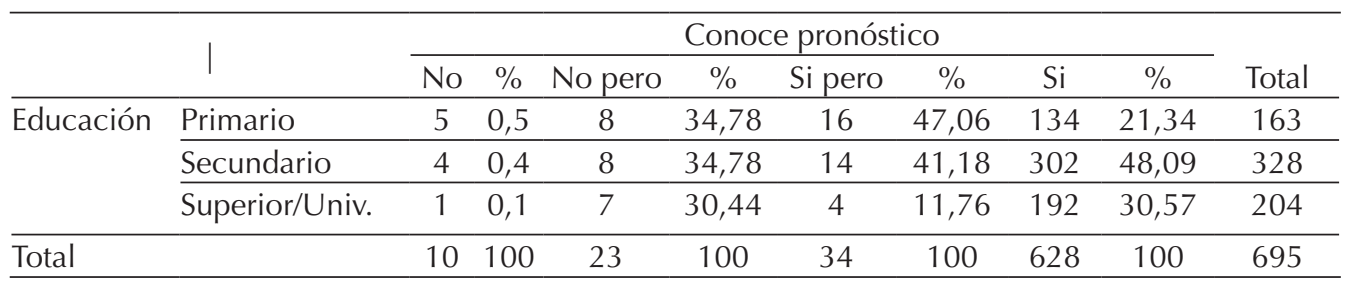

todo respecto a la toxicidad o efectos adversos y el resto quería conocer algún aspecto de la toxicidad (por ejemplo la toxicidad más severa).

Cuando se interrogó acerca de la necesidad de obtener conocimientos adicionales, se evidenció que 710 pacientes $(86,6 \%)$ se mostraron interesados en conocer sobre cuidados paliativos, $19(2,3 \%)$ no estaban interesados y $91(11,1 \%)$ se abstuvieron de responder.

Sumado a lo anterior, 676 participantes $(82,4 \%)$ se mostraron interesados en conocer sobre investigación clínica, 48 (5,8\%) no estaban interesados y $96(11,7 \%)$ se abstuvieron de responder.

Adicionalmente, 611 pacientes $(74,5 \%)$ se mostraron interesados en conocer a miembros del equipo de psico-oncología, $111(13,5 \%)$ no estaban interesados y 98 $(12 \%)$ no respondieron acerca de esta consulta. En cambio, 487 sujetos $(59,4 \%)$ declararon estar interesados en recibir soporte espiritual, $206(25,1 \%)$ no estaban interesados y 127 (15,5\%) decidieron no responder.

En este punto se observó una clara diferencia entre los sexos, dado que, si bien los dos grupos estaban igualmente interesados en Investigación y en cuidados paliativos, las mujeres se mostraron más interesadas que los varones en el soporte psicológico $\left(X^{2}=8,035 ; p=0,005\right)$ y espiritual $\left(X^{2}=4,182 ; p=0,041\right)$. Asimismo, los participantes con secundario completo presentaron un interés significativamente mayor por la investigación $\left(X^{2}=14,987\right.$; $\mathrm{p}=0,001)$. Sumado a lo anterior, los pacientes de un rango etario de 50-70 años también presentaron un interés significativamente mayor por la investigación $\left(X^{2}=\right.$ $8,751 ; p=0,033)$.

Lugar en donde prefieren fallecer

Con respecto al lugar del fallecimiento, 331 pacientes $(40,4 \%)$ prefirieron el sanatorio, $260(31,7 \%)$ el domicilio y 229 $(27,9 \%)$ no contestaron. Es importante reportar en este sentido que la única variable que se relacionó con esta elección, si bien no de manera estadísticamente significativa $(p=0,07)$, fue el estadio de enfermedad que pudo estudiarse en 470 pacientes: Ios pacientes Estadio I y II tuvieron una mayor 
tasa de no respuesta y/o elección del sanatorio como lugar de fallecimiento que los estadios III-IV. Planteando que probablemente el tiempo óptimo de análisis de esta problemática no sea inmediatamente después del diagnóstico de una enfermedad potencialmente curable.

\section{Características valoradas del equipo médico}

Las condiciones más valoradas en el médico fueron: conocimiento (432 pacientes, $52,7 \%$ ), honestidad (373 pacientes, 45,5\%), humanidad (363 pacientes, 44.3\%), disponibilidad (270 participantes, 32,9\%), experiencia (266 pacientes, 32,4\%), humildad (160 participantes, 19,5\%), simpatía (154 participantes, $18,8 \%$ ) y otras (25 participantes, 3\%). Sin embargo en este punto hubo una diferencia entre los sexos: aunque para ambos grupos lo más importante era el conocimiento, las mujeres antepusieron la humanidad $\left(X^{2}=15,785 ; \mathrm{p}<0,001\right) \mathrm{a}$ la honestidad (Ver tabla 8), mientras que los varones antepusieron la honestidad y la experiencia a la humanidad como valores deseables en el médico.

\section{Opinión sobre la utilidad de la encuesta}

Finalmente, se les solicitó a los participantes que calificaran la encuesta: 675 pacientes $(82,3 \%)$ la consideraron Buena, Muy Buena o Excelente. Cuatrocientos setenta y seis pacientes (58\%) la consideraron útil, $283(34,5 \%)$ la evaluaron como necesaria y $194(23,7 \%)$ la calificaron como recomendable.

\section{DISCUSIÓN}

El presente estudio representa, en nuestro conocimiento, el primer intento en nuestro medio por identificar las necesidades y los deseos de los pacientes por conocer cuestiones vinculadas con su enfermedad.

Los resultados obtenidos plantean que los pacientes, en su gran mayoría, conocen su diagnóstico, desean conocer su pronóstico, requieren mayor información sobre la utilidad y la toxicidad de los tratamientos, pueden analizar la elección de un familiar que represente su voluntad al final de la vida y la mayoría considera la internación hospitalaria como el mejor lugar para morir. Además, todos ellos valorarían contar con información sobre cuidados paliativos e investigación clínica.

Sumado a lo anterior, las mujeres se muestran más propensas a recibir ayuda psicológica y espiritual.

En cuanto a los valores reconocidos en un profesional, el valor preferido por los pacientes fue el conocimiento, seguido por la humanidad en el trato, la honestidad y la experiencia.

Por otro lado, hemos encontrado respuestas que han puesto en discusión nuestras expectativas previas. Por un lado hemos observado que, si bien la gran mayoría de los encuestados ha valorado positivamente la encuesta, un gran porcentaje no la recomendaría. Esta cuestión podría hacernos pensar que, si bien los encuestados valoran positivamente el diseño y el contenido, movilizaría cuestiones personales que preferirían que otros evitaran.

\section{Tabla 8. Característica valorada en el Médico Tratante (Humanidad).}

\begin{tabular}{ccccccc}
\hline & & \multicolumn{4}{c}{ Humanidad en el Médico } & \\
\cline { 3 - 6 } & & No & $\%$ & Si & $\%$ & Total \\
\hline \multirow{3}{*}{ Sexo } & Femenino & 160 & 54,05 & 172 & 70,78 & 332 \\
& Masculino & 136 & 45,95 & 71 & 29,22 & 207 \\
& Total & 296 & 100 & 243 & 100 & 539 \\
\hline
\end{tabular}


Por otro lado hemos observado una búsqueda deliberada de participación activa en las decisiones vinculadas al manejo de la información y los tratamientos.

Finalmente hubo un alto porcentaje de encuestados que no han respondido sobre el lugar de fallecimiento. En estudios posteriores deberíamos evaluar si esto se debe a eludir de la respuesta o al hecho de que en algunos casos los encuestados ni piensan en esa posibilidad al momento de ser encuestados, dependiendo esta cuestión del estadio de la enfermedad y del diagnóstico oncológico.

En cuanto a las limitaciones del estudio podríamos pensar que, en el afán por abarcar distintos temas de interés, la encuesta ha sido prolongada y pudo haber desalentado la participación. Asimismo, una encuesta con menos alternativas podría ser mejor y podría ayudar en la comprensión de la misma.

\section{REFERENCIAS BIBLIOGRÁFICAS}

1. Lamont EB, Christakis NA. Prognostic disclosure to patients with cancer near the end of life. Ann Intern Med 2001;134: 1096-105. Doi: 10.7326/0003-4819-13412-200106190-00009

2. Christakis N. Death Foretold: Prophecy and prognosis in medical care. Chicago: University of Chicago Press; 1999.

3. Leydon GM, Boulton M, Moynihan C, Jones A, Mossman J, Boudioni $M$, et al Cancer patients' information needs and information seeking behavior: In depth interview study. BMJ 2000;320:909-13. Doi: Doi:10.1136/bmj.320.7239.909

4. Hagerty RG, Butow PN, Ellis PA, Lobb EA, Pendlebury S, Leighl N, et al. Cancer patient preferences for communication of prognosis in the metastatic setting. J Clin Oncol 2004;22:1721-30. Doi: 10.1200/ JCO.2004.04.095
5. Cherlin E, FriedT, Prigerson HG, SchulmanGreen D, Johnson-Hurzeler R, Bradley EH. Communication between physicians and family caregivers about care at the end of life: when do discussions occur and what is said? J Palliat Med 2005;8: 1176-85. Doi: 10.1089/jpm.2005.8.1176

6. Helft PR. Necessary collusion: Prognostic communication with advanced cancer patients. J Clin Oncol 2005;23:3146-50. Doi: 10.1200/JCO.2005.07.003

7. Selman L, Harding R, Beynon $T$, et al. Improving end-of-life-care for patients with chronic heart failure: "Let's hope it'll get better, when I know in my heart of hearts it won't. Heart 2007;93:963-7. Doi:10.1136/hrt.2006.106518

8. Meier DE, Back AL, Morrison RS. The inner life of physicians and care of the seriously ill. JAMA 2001;286:3007-14. Doi:10.1001/jama.286.23.3007

9. Wright AA, Zhang B, Ray A, Mack JW, Trice E, Balboni $\mathrm{T}$, et al. Associations between end-of-life discussions, patient mental health, medical care near death, and caregiver bereavement adjustment. JAMA 2008;300:1665-73. Doi:10.1001/ jama.300.14.1665.

10. SmithTJ, Dow LA, Virago EA, Khatcheressian J, Matsuyama R and Lyckholm LJ. A pilot trial of decision aids to give truthful prognostic and treatment information to chemotherapy patients with advanced cancer. J Support Oncol 2011;9:79-86. Doi:10.1016/j.suponc.2010.12.005

11. Kirk P, Kirk I, Kristjanson LJ. What do patients receiving palliative care for cancer and their families want to be told? A Canadian and Australian qualitative study. BMJ 2004; 328: 1343. Doi:10.1136/ bmj.38103.423576.55

12. Emanuel LL, Ferris FD, von Gunten CF, Von Roenn J. EPEC-O: Education in palliative and end of life care for oncology, Chicago, EPEC Project, 2005. 


\section{Anexo
ENCUESTA SOBRE ASPECTOS ÉTICOS, NECESIDADES Y DIRECTIVAS ANTICIPADAS DE LOS PACIENTES}

\section{DATOS PERSONALES*}

Nombre y Apellido:

Fecha de Nacimiento: DNI:

Estado Civil: Hijos:

Nivel educativo: $\square$ Primaria incompleta

口 Secundaria incompleta

- Primaria completa

口 Terciaria incompleta

- Secundaria completa

口 Universitario incompleto

a Terciaria completa

口 Universitario completo

Situación laboral:

$\square$ Desempleado

Trabajador activo

口 Jubilado

Es la primera vez que concurro al instituto

$$
\text { 口 } \mathrm{Si} \quad \square \quad \text { No. }
$$

Conoce su diagnóstico

$$
\text { 口 } \mathrm{Si} \quad \mathrm{No} \text {. }
$$

Si No conoce su diagnóstico, conoce el motivo por el cual fue derivado a nuestra institución.

$$
\text { 口 Si } \quad \text { No. }
$$

En que medida considera que conoce ese motivo

$$
\text { 口 Mucho } \square \text { Poco }
$$

Alguna vez realizó quimioterapia

$$
\text { 口 } \mathrm{Si} \quad \square \mathrm{No} \text {. }
$$

* Si bien usted nos provee sus datos personales, a los efectos del análisis y de una eventual publicación científica, estos datos serán reemplazados por una clave con la finalidad de que no se puedan relacionar con ninguna persona. 


\section{ASPECTOS ÉtICOS*}

Las siguientes preguntas sobre valores y deseos personales están orientadas a conocer lo que usted quiere saber o no, acerca de su enfermedad y los eventuales tratamientos.

1. En el caso de que tenga una enfermedad maligna / grave deseo que el médico me informe el diagnóstico

$$
\text { 口 } \mathrm{Si} \quad \square \quad \mathrm{No} \text {. }
$$

2. En el caso de que haya seleccionado No ¿Hay alguien a quien usted desea que se le informe el diagnóstico?

$$
\text { 口 Si } \quad \text { No. }
$$

3. Si desea que le informemos a alguien por favor indíquenos en quien deposita esa confianza.

Nombre: NO LLENAR

Parentesco:

Dirección: NO LLENAR

Teléfono: NO LLENAR

4. En el caso de que un familiar suyo desee hablar con el médico sobre su caso o ver la historia clínica ¿Usted lo autoriza?
口 $\mathrm{Si}$
№.

5. Si lo/s autoriza por favor indíquenos el nombre de cada uno de ellos nombres autorizados a ver mi historia clínica y hablar con el médico:

NO LLENAR

\section{NECESIDADES* DE LOS PACIENTES}

Cada uno de los pacientes tiene distintas necesidades de información médica para poder decidir sobre el futuro de su vida.

6. ¿A usted le interesaría conocer el pronóstico* de su enfermedad?

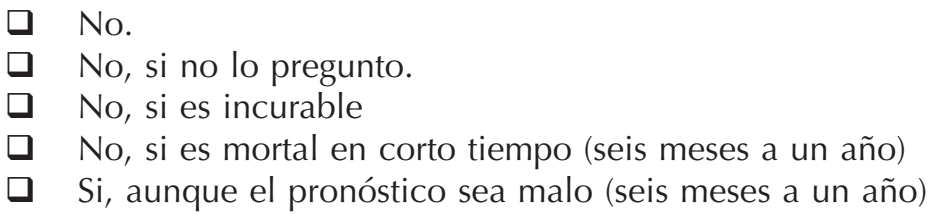




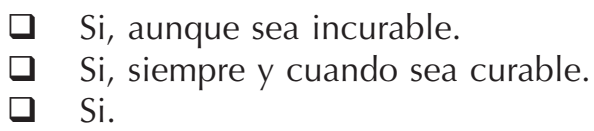

7. En el caso de los tratamientos ¿Qué es lo que Ud. necesita conocer sobre la utilidad* del tratamiento?

- Sólo necesito saber si me puede curar o no.

- Necesito saber además si las chances de curarme son muchas o pocas.

- Si no pudiera curarme necesito saber si puede alargar mi vida en semanas $\square$, meses $\square$ o años $\square$.

- Si no pudiera alargar mi vida necesito saber si puede aliviar mis síntomas.

口 Necesito saber todo lo que el tratamiento puede hacer para beneficiarme

- Necesito saber exclusivamente lo que el médico quiera decirme.

口 No necesito saber nada.

8. En el caso de la toxicidad* de un tratamiento ¿Qué es lo que Ud. necesita conocer?

- Sólo necesito saber si me puedo morir o no debido al tratamiento.

- Necesito saber además cuales son las chances de que pueda morir por el tratamiento (muchas o pocas).

- Necesito saber los principales efectos indeseables.

- Necesito saber todos los efectos indeseables.

- Necesito saber sólo lo que el médico quiera decirme.

ㄱo necesito saber nada.

9. En el caso de que no hubiera un tratamiento comprobado para su enfermedad ¿Desearía conocer alternativas de investigación* para el tratamiento de la enfermedad?

$$
\text { 口 Si a No. }
$$

10.En el caso de que la enfermedad empeore y le provoque síntomas de difícil control ¿Desearía conocer a un equipo de cuidados paliativos* (alivio de síntomas?)
$\square \mathrm{Si}$
№.

11.En el caso de que Usted sea una persona religiosa ¿Desearía tener la oportunidad de hablar sobre los aspectos espirituales de su vida con un asesor espiritual*?

$$
\square \mathrm{Si} \quad \square \quad \text { No. }
$$

12. En el caso de que Usted considere útil entrevistarse con profesionales del área psicológica* ¿Desearía tener la oportunidad de hablar con alguno de los profesionales del equipo?

口 $\mathrm{Si} \quad \square \quad \mathrm{No}$.




\section{DIRECTIVAS ANTICIPADAS*}

Estas preguntas nos permitirán conocer cuál es su deseo sobre temas muy delicados para la vida y la muerte de una persona.

13. En el caso de que en algún momento futuro debido al empeoramiento de la enfermedad o por razones ajenas a ella usted perdiera la capacidad* de tomar decisiones ¿A quién desearía que consultemos sobre las decisiones médicas a tomar?

Nombre: NO LLENAR

Parentesco:

Dirección: NO LLENAR

Teléfono: NO LLENAR

14. En el caso de que la enfermedad no se pueda controlar y lamentablemente le cause la muerte, muchos pacientes días o semanas antes de morir son internados en hospitales, clínicas o sanatorios, mientras que otros pacientes desean morir en sus casas. ¿Cuál sería su deseo para ese momento?

- Fallecer en el sanatorio $\square \quad$ Fallecer en el domicilio

\section{OBSERVACIONES}

Buenos Aires

de de

Firma:

Aclaración:

Número de documento:

\section{EVALUACIÓN DE LA ENCUESTA}

1. Usted considera que la encuesta es (seleccione todas las que desee):
a.
$\square \quad$ Util
b.
a Clara
$\square$ Inútil
c. $\square$ Completa
口 Confusa
d. $\square$ Profunda
a Incompleta
e. $\square$ Precisa
$\square$ Superficial
f. $\square$ Necesaria
ㅁ Extensa
g. $\square$ Recomendable
Innecesaria
$\square \quad$ No es recomendable 
2. Si tuviera que calificar la encuesta la consideraría:

$\begin{array}{cl}\square & \text { Muy mala } \\ \square & \text { Mala } \\ \square & \text { Regular } \\ \square & \text { Buena } \\ \square & \text { Muy buena } \\ \square & \text { Excelente }\end{array}$

3. Las siguientes características son importantes en un médico, pero para Ud. las tres más importantes son (márquelas con un círculo):
a. Conocimientos científicos (que esté actualizado)
b. Experiencia (que tenga varios años de profesión)
c. Honestidad (que le diga siempre lo que piensa)
d. Simpatía (que lo trate amablemente)
e. Disponibilidad (que esté cuando usted lo necesita)
f. Humildad (si no sabe que consulte con otros)
g. Humanidad (que le hable con cariño y respeto)
h. Otra/s:

4. A Usted le parece que en la encuesta falta preguntar sobre el/los siguientes temas o bien desea hacer un comentario: 
